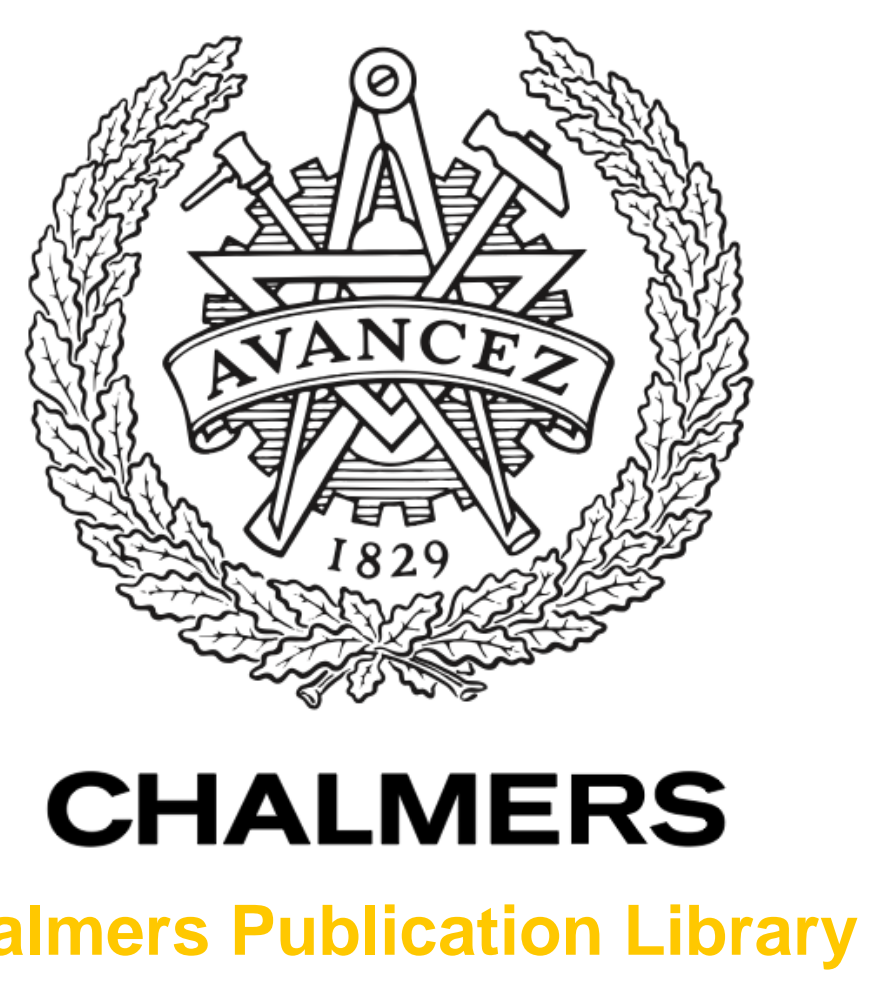

Chalmers Publication Library

\title{
Coordinated Hybrid Automatic Repeat Request
}

This document has been downloaded from Chalmers Publication Library (CPL). It is the author's version of a work that was accepted for publication in:

IEEE Communications Letters (ISSN: 1089-7798)

Citation for the published paper:

Makki, B. ; Svensson, T. ; Eriksson, T. et al. (2014) "Coordinated Hybrid Automatic Repeat Request". IEEE Communications Letters, vol. 18(11), pp. 1975-1978.

http://dx.doi.org/10.1109/LCOMM.2014.2360178

Downloaded from: http://publications.lib.chalmers.se/publication/202707

Notice: Changes introduced as a result of publishing processes such as copy-editing and formatting may not be reflected in this document. For a definitive version of this work, please refer to the published source. Please note that access to the published version might require a subscription.

Chalmers Publication Library (CPL) offers the possibility of retrieving research publications produced at Chalmers University of Technology. It covers all types of publications: articles, dissertations, licentiate theses, masters theses, conference papers, reports etc. Since 2006 it is the official tool for Chalmers official publication statistics. To ensure that Chalmers research results are disseminated as widely as possible, an Open Access Policy has been adopted.

The CPL service is administrated and maintained by Chalmers Library. 


\title{
Adaptive Space-Time Coding using ARQ
}

\author{
Behrooz Makki, Tommy Svensson, Thomas Eriksson and Mohamed-Slim Alouini, Fellow, IEEE
}

\begin{abstract}
We study the energy-limited outage probability of the block space-time coding (STC)-based systems utilizing automatic repeat request (ARQ) feedback and adaptive power allocation. Taking the ARQ feedback costs into account, we derive closed-form solutions for the energy-limited optimal power allocation and investigate the diversity gain of different STCARQ schemes. Also, sufficient conditions are derived for the usefulness of ARQ, in terms of energy-limited outage probability. The results show that, for a large range of feedback costs, the energy efficiency is substantially improved by the combination of ARQ and STC techniques, if optimal power allocation is utilized.
\end{abstract}

\section{INTRODUCTION}

Automatic repeat request (ARQ) techniques are commonly used in wireless networks to combat the loss of data packets due to channel fading. In ARQ schemes, if the receiver fails in decoding the data correctly, it asks for a retransmission. Then, depending on the data retransmission approach, different types of ARQ are defined in the literature [1]-[8]. Type I is the simplest version of ARQ, where both the error-detecting and the forward error correction information are added to each message and the receiver disregards the previous messages, if received in error. In Type II (resp. Type III), a new (resp. the same) data is sent in the retransmission rounds and, in each round, the receiver combines all signals received up to the end of that round.

With multiple antennas, an alternative for Types I-III ARQ is to use the space-time coding (STC) techniques in an ARQ-based fashion. With a STC-based ARQ approach [9]-[14], a permuted version of the initial sub-codeword is sent in the retransmissions and, in each round, the receiver combines all received permutations of the initial signal for message decoding. Thus, the STC-based ARQ is an intermediate type of ARQ with (almost) the same complexity as in Type III and data transmission efficiency comparable with Type II ARQ [9]-[14]. Moreover, the implementation of STC-ARQ protocols is of interest when we remember that the STCs are among the best approaches for exploiting the spatial diversity and are considered in different standards, e.g., [15]. Then, as shown in the following, the performance of the STC-based setups is improved substantially, if they are combined with ARQ. These are the main motivations for this correspondence, in which we analyze the performance of STC-ARQ systems using adaptive power allocation ${ }^{1}$.

Copyright (c) 2013 IEEE. Personal use of this material is permitted. However, permission to use this material for any other purposes must be obtained from the IEEE by sending a request to pubs-permissions@ieee.org.

B. Makki, T. Svensson and T. Eriksson are with Chalmers University of Technology, Email: \{behrooz.makki, tommy.svensson, thomase\}@chalmers.se. M.-S. Alouini is with the King Abdullah University of Science and Technology (KAUST), Email: slim.alouini@kaust.edu.sa

${ }^{1}$ Here, we study the fixed-length coding schemes. Thus, as illustrated in the following, adaptive energy allocation is achieved by updating the sub-codewords powers which are the sub-codewords energies scaled by a constant (length of the sub-codewords). 
Adaptive power/energy allocation in Types I-III ARQ protocols is addressed in many papers, e.g., [1]-[8]. Moreover, the combination of ARQ and different STCs is studied in, e.g., [9]-[14], where the results are obtained with uniform (non-adaptive) power allocation.

In this correspondence, we study the problem of energy-limited outage probability minimization for data transmission in block STC-ARQ protocols. The contributions of the paper are three folds:

- We derive closed-form solutions for the outage-limited optimal power allocation in different STC-ARQ schemes and show substantial energy efficiency improvement via optimal power allocation in STC-ARQ protocols. For instance, consider the antenna switching (AS), the Alamouti, the spatial multiplexing with repetition (SMR) and the cyclic delay diversity (CDD) kinds of STC with a codeword rate 1 nats-per-channel-use (npcu) and outage probability $10^{-4}$. Then, compared to the STC approach, the implementation of STC-ARQ improves the energy efficiency by 6.4, 8.7, 10.8 and $9.3 \mathrm{~dB}$, respectively.

- We analyze the moderate/high signal-to-noise ratio (SNR) performance of the STC-ARQ protocols; we show that the diversity gain of the AS, the Alamouti, the SMR and the CDD kinds of STC-ARQ increases from 2 without ARQ to 3, 6, 6 and 6, respectively, if they are combined with ARQ and the transmission powers are optimally allocated between the retransmissions.

- For different STCs, we find sufficient conditions for the usefulness of ARQ, in terms of energy-limited outage probability. Specially, we show the combination of STC and ARQ techniques to be considerably useful for a large range of feedback costs.

Notations. The probability density function (pdf) of the random variable $\Delta$ is denoted by $f_{\Delta}$. Also, $\operatorname{det}(\mathbf{X}), \mathbf{X}^{*}, \mathbf{X}^{\mathrm{T}}$ and $\mathbf{X}^{\mathrm{h}}$ are the determinant, the conjugate, the transpose and the Hermitian of the matrix $\mathbf{X}$, respectively.

\section{PROBlem FORMULATION}

Consider a $2 \times 1$ multiple-input-single-output (MISO) setup utilizing STC. With the STC, the mother codeword, of length $2 L$ channel uses, can be represented as $\mathbf{X}=\left[\begin{array}{ll}\mathbf{x}_{11} & \mathbf{x}_{12} \\ \mathbf{x}_{21} & \mathbf{x}_{22}\end{array}\right] \in \mathcal{C}^{2 \times 2 L}$ where the sub-codeword $\mathbf{X}_{2}=\left[\mathbf{x}_{12}^{\mathrm{T}} \mathbf{x}_{22}^{\mathrm{T}}\right]^{\mathrm{T}} \in \mathcal{C}^{2 \times L}$ is a permuted representation of the initial sub-codeword $\mathbf{X}_{1}=\left[\begin{array}{ll}\mathbf{x}_{11}^{\mathrm{T}} & \mathbf{x}_{21}^{\mathrm{T}}\end{array}\right]^{\mathrm{T}} \in \mathcal{C}^{2 \times L}$. Considering $n$ information nats for the sub-codeword $\mathbf{X}_{1}$ and because $\mathbf{X}_{2}$ is a permuted representation of $\mathbf{X}_{1}$, the codeword rate is given by $R=\frac{n}{2 L} \mathrm{npcu}$. The codeword can be sent in two ways as follows.

Scenario 1 (STC-ARQ): Using the ARQ-based STC, the data is sent in two rounds; first, $\mathbf{X}_{1}$ is sent via $L$ channel uses. Thus, the received signal is

$$
\mathbf{Y}_{1}=\left[h_{1} h_{2}\right] \mathbf{X}_{1}+\mathbf{Z}_{1},
$$

where $\mathbf{Z}_{1} \in \mathcal{C}^{1 \times L}$ is the independent and identically distributed (iid) complex Gaussian noise matrix whose elements follow $\mathcal{C N}(0,1)$ and $\mathbf{H}=\left[h_{1} h_{2}\right]$ represents the fading matrix. 
Let us denote the maximum achievable rate of the channel model (1) by $C_{1}$ and, in harmony with, e.g., [5]-[10], assume the codewords to be Gaussian and sufficiently long (see [1] for discussions on the length of the ARQ subcodewords). If $C_{1} \geq \frac{n}{L}=2 R$, the data is correctly decoded by the receiver, a positive acknowledgement (ACK) is fed back to the transmitter and the data retransmission stops. Thus, in this case, the consumed energy of the forward link is $\psi_{1}=\phi_{1} L$ where $\phi_{i}$ is the power of the sub-codeword $\mathbf{X}_{i}$. Otherwise, if $C_{1}<2 R$, the receiver sends a negative acknowledgement (NACK) and asks for a retransmission. Hence, $L$ more channel uses are used to send $\mathbf{X}_{2}$ and the total consumed energy in the forward link increases to $\left(\phi_{1}+\phi_{2}\right) L$. In this way, the total weighted energy of the ARQ-based approach is obtained by

$$
\begin{aligned}
\Psi^{\mathrm{STC}-\mathrm{ARQ} Q} & =\phi_{1} L \operatorname{Pr}\left(C_{1} \geq 2 R\right)+\left(\phi_{1} L+\phi_{2} L\right) \operatorname{Pr}\left(C_{1}<2 R\right)+w \psi^{\mathrm{f}} \\
& =\phi_{1} L+\phi_{2} L \operatorname{Pr}\left(C_{1}<2 R\right)+w \psi^{\mathrm{f}} .
\end{aligned}
$$

Here, $\psi^{\mathrm{f}}$ is the energy consumed by the transmitter and the receiver during the feedback process and $w$ is a weighting factor motivated by the fact that the transmitter and the receiver have different power supplies, etc. Also, (2) is based on the fact that, independently of the message decoding status, an ARQ feedback signal (resp. no feedback) is sent at the end of the first (resp. the second) round.

Sending $\mathbf{X}_{2}$, the receiver combines the received signals of the two rounds. Hence, at the end of the second round, the equivalent channel model is changed to

$$
\mathbf{Y}_{2}=\left[h_{1} h_{2}\right]\left[\mathbf{X}_{1} \mathbf{X}_{2}\right]+\mathbf{Z}_{2}, \mathbf{Z}_{2} \sim \mathcal{C N}^{2 \times 2 L} .
$$

Denoting the maximum achievable rate of the channel model (3) by $C_{2}$, the data is correctly decoded (resp. outage occurs) if $C_{2} \geq \frac{n}{2 L}=R$ (resp. $C_{2}<R$ ). Thus, the outage probability of the STC-ARQ based approach is $\operatorname{Pr}\left(C_{2}<R\right)$ and, for a given feedback cost $\psi^{\mathrm{f}}$ and codeword rate $R$, the energy-limited outage minimization problem is rephrased as

$$
\left\{\begin{array}{l}
\min _{\phi_{1}, \phi_{2}} \operatorname{Pr}\left(C_{2}<R\right) \\
\text { s.t. } \phi_{1}+\phi_{2} \operatorname{Pr}\left(C_{1}<2 R\right) \leq \bar{\phi}^{\text {STC-ARQ }}, \\
\bar{\phi}^{\text {STC-ARQ }} \doteq \frac{\bar{\psi}-Q^{\mathrm{f}} L}{L}, Q^{\mathrm{f}} \doteq \frac{w \psi^{\mathrm{f}}}{L}
\end{array}\right.
$$

Here, $\bar{\psi}$ is the total energy budget, $Q^{\mathrm{f}} \doteq \frac{w \psi^{\mathrm{f}}}{L}$ denotes the relative feedback cost and (4.ii) follows from (2).

Scenario 2 (STC): With the STC, which we consider as the baseline, $2 L$ channel uses are used to send the whole codeword in one shot. Thus, the channel model is the same as the one in (3) and the outage probability is $\operatorname{Pr}\left(C_{2}<R\right)$, the same as in the ARQ-based scheme. Then, as there is no feedback and the whole codeword is sent in one round, the total consumed energy is found as $\left(\phi_{1}+\phi_{2}\right) L$ which rephrases the energy-limited outage minimization problem as

$$
\left\{\begin{array}{l}
\min _{\phi_{1}, \phi_{2}} \operatorname{Pr}\left(C_{2}<R\right), \\
\text { s.t. }\left(\phi_{1}+\phi_{2}\right) \leq \bar{\phi}, \bar{\phi} \doteq \frac{\bar{\psi}}{L} .
\end{array}\right.
$$


Intuitively, (4) and (5) mean that with the ARQ we do gambling; first, half of the STC-based codeword is sent. If the channel quality is high, the data is correctly decoded at the end of the first round and the energy cost for the second run is saved. Otherwise, the sub-codeword $\mathbf{X}_{2}$ is sent, the same as in the non-ARQ approach. The cost of the gambling is the cost for feedback, i.e., $\psi^{\mathrm{f}}$. Thus, depending on the feedback cost, the implementation of ARQ might or might not improve the performance of the STC-based schemes. In the following, we study (4) and (5) for different kinds of STC.

\section{Performance ANALysis For DifFERENT STCs}

This section analyzes the energy-limited outage minimization problem for the AS, the Alamouti, the SMR and the CDD kinds of STC (See Section I). First, the probabilities $\operatorname{Pr}\left(C_{1}<2 R\right)$ and $\operatorname{Pr}\left(C_{2}<R\right)$ are derived for the considered STCs and then (4) and (5) are solved for each one. Note that the considered STCs are only examples and, as illustrated in Subsection III.B, the same techniques are applicable for the other kinds of STC/number of antennas.

To calculate the probabilities, we need to find the achievable rate terms $C_{1}$ and $C_{2}$ for each scheme. While these terms are summarized in Table 1, because of the mathematical similarity, we only explain the procedure for deriving the achievable rate terms of the CDD code.

Using the CDD, the mother codeword is given by $\mathbf{X}^{\mathrm{CDD}}=\left[\begin{array}{c}\sqrt{\frac{\phi_{1}}{2}} \mathbf{u} \sqrt{\frac{\phi_{2}}{2}} \mathbf{v} \\ \sqrt{\frac{\phi_{1}}{2}} \mathbf{v} \sqrt{\frac{\phi_{2}}{2}} \mathbf{u}\end{array}\right], \frac{1}{L} \sum_{l=1}^{L}|\mathbf{u}[l]|^{2} \leq 1, \frac{1}{L} \sum_{l=1}^{L}|\mathbf{v}[l]|^{2} \leq 1$. Thus, the channel models in the first and the second rounds, i.e., (1) and (3), are

$$
\begin{aligned}
& \mathbf{Y}_{1}^{\mathrm{CDD}}=\tilde{\mathbf{H}}_{1}^{\mathrm{CDD}}[\mathbf{u} \mathbf{v}]^{\mathrm{T}}+\mathbf{Z}_{1}, \tilde{\mathbf{H}}_{1}^{\mathrm{CDD}} \doteq \sqrt{\frac{\phi_{1}}{2}}\left[h_{1} h_{2}\right], \\
& \mathbf{Y}_{2}^{\mathrm{CDD}}=\left[h_{1} h_{2}\right] \mathbf{X}^{\mathrm{CDD}}+\mathbf{Z}_{2} \equiv \tilde{\mathbf{H}}_{2}^{\mathrm{CDD}}[\mathbf{u} \mathbf{v}]^{\mathrm{T}}+\mathbf{Z}_{2}, \tilde{\mathbf{H}}_{2}^{\mathrm{CDD}} \doteq\left[\begin{array}{cc}
\sqrt{\frac{\phi_{1}}{2}} h_{1} & \sqrt{\frac{\phi_{1}}{2}} h_{2} \\
\sqrt{\frac{\phi_{2}}{2}} h_{2} & \sqrt{\frac{\phi_{2}}{2}} h_{1}
\end{array}\right],
\end{aligned}
$$

where $\tilde{\mathbf{H}}_{i}$ denotes the equivalent channel model of a scheme at the end of round $i$. In this way, we use the capacity of the multiple-input-multiple-output (MIMO) setups [16] to find the maximum achievable rates as

$$
\begin{gathered}
C_{1}^{\mathrm{CDD}}=\log \left(1+\tilde{\mathbf{H}}_{1}^{\mathrm{CDD}}\left(\tilde{\mathbf{H}}_{1}^{\mathrm{CDD}}\right)^{\mathrm{h}}\right)=\log \left(1+\frac{\phi_{1}}{2} G\right), G \doteq g_{1}+g_{2}, g_{i}=\left|h_{i}\right|^{2}, i=1,2, \\
C_{2}^{\mathrm{CDD}}=\frac{1}{2} \log \operatorname{det}\left(\mathbf{I}_{2}+\tilde{\mathbf{H}}_{2}^{\mathrm{CDD}}\left(\tilde{\mathbf{H}}_{2}^{\mathrm{CDD}}\right)^{\mathrm{h}}\right)=\frac{1}{2} \log \operatorname{det}\left(\mathbf{I}_{2}+\left[\begin{array}{cc}
\frac{\phi_{1}}{2} G & \sqrt{\phi_{1} \phi_{2}} \operatorname{Re}\left\{h_{1} h_{2}^{*}\right\} \\
\sqrt{\phi_{1} \phi_{2}} \operatorname{Re}\left\{h_{1} h_{2}^{*}\right\} & \frac{\phi_{2}}{2} G
\end{array}\right]\right) \\
=\frac{1}{2} \log \left(\left(1+\frac{\phi_{1}}{2} G\right)\left(1+\frac{\phi_{2}}{2} G\right)-\phi_{1} \phi_{2} \operatorname{Re}\left\{h_{1} h_{2}^{*}\right\}^{2}\right),
\end{gathered}
$$

where $\mathbf{I}_{m}$ denotes the $m \times m$ identity matrix. Note that for Rayleigh fading channels $h_{i} \sim \mathcal{C N}(0,1), \forall i$, on which we focus, the pdf of random variables $g_{i}=\left|h_{i}\right|^{2}, i=1,2$, and $G=g_{1}+g_{2}$ are given by $f_{g_{i}}(g)=e^{-g}$ and $f_{G}(g)=g e^{-g}, g \geq 0$, respectively. Also, the achievable rate terms of the other STCs considered in Table 1 are obtained with the same procedure as in (6)-(8).

Using Table 1, the probabilities $\operatorname{Pr}\left(C_{1}<2 R\right)$ and $\operatorname{Pr}\left(C_{2}<R\right)$ are obtained for different STCs as follows.

AS-based STC-ARQ: 
Table I

THE EQUIVALENT CHANNEL MODELS AND THE ACHIEVABLE RATES OF DIFFERENT STC-ARQ SCHEMES.

\begin{tabular}{|c|c|c|c|c|c|}
\hline \multicolumn{2}{|c|}{ Mother STC metho } & $\tilde{\mathbf{H}}_{1}$ & $C_{1}$ & $\tilde{\mathbf{H}}_{2}$ & $C_{2}$ \\
\hline A & $\begin{array}{l}\sqrt{\phi_{1}} \mathbf{u} 0 \\
0 \sqrt{\phi_{2}} \mathbf{u}\end{array}$ & $\sqrt{\phi_{1}}\left[h_{1} 0\right]$ & $\log \left(1+\phi_{1} g_{1}\right)$ & {$\left[\sqrt{\phi_{1}} h_{1} \sqrt{\phi_{2}} h_{2}\right]$} & $\frac{1}{2} \log \left(1+\phi_{1} g_{1}+\phi_{2} g_{2}\right)$ \\
\hline SMR: & $\sqrt{\frac{\phi_{1}}{2}} \mathbf{v} \sqrt{ }$ & $\sqrt{\frac{\phi_{1}}{2}}\left[h_{1} h_{2}\right]$ & $\log \left(1+\frac{\phi_{1}}{2}\left(g_{1}+g_{2}\right)\right)$ & $\sqrt{\frac{\phi_{2}}{2}} h_{1} \sqrt{ }$ & $\frac{1}{2} \log \left(1+\left(\frac{\phi_{1}}{2}+\frac{\phi_{2}}{2}\right)\left(g_{1}+g_{2}\right)\right)$ \\
\hline Alamouti: & $\begin{array}{l}\sqrt{\frac{\phi_{1}}{2} \mathbf{u}} \\
\sqrt{\frac{\phi_{1}}{2} \mathbf{v}} \\
\end{array}$ & $\sqrt{\frac{\phi_{1}}{2}}\left[h_{1} h_{2}\right]$ & $\log \left(1+\frac{\phi_{1}}{2}\left(g_{1}+g_{2}\right)\right)$ & $\begin{array}{ll}\sqrt{\frac{\phi_{1}}{2}} h_{1} & \sqrt{\frac{\phi_{1}}{2}} h_{2} \\
\sqrt{\frac{\phi_{2}}{2}} h_{2}^{*} & -\sqrt{\frac{\phi_{2}}{2}} h_{1}^{*}\end{array}$ & $\frac{1}{2} \sum_{i=1}^{2} \log \left(1+\frac{\phi_{i}}{2}\left(g_{1}+g_{2}\right)\right)$ \\
\hline CDD: & $\begin{array}{l}\sqrt{\frac{\phi_{1}}{2}} \mathbf{u} \\
\sqrt{\frac{\phi_{1}}{2}} \mathbf{v} \sqrt{ }\end{array}$ & $\sqrt{\frac{\phi_{1}}{2}}\left[h_{1} h_{2}\right]$ & $\log \left(1+\frac{\phi_{1}}{2}\left(g_{1}+g_{2}\right)\right)$ & {$\left[\begin{array}{cc}\sqrt{\frac{\varphi_{1}}{2}} h_{1} & \sqrt{\frac{\varphi_{1}}{2}} h_{2} \\
\sqrt{\frac{\phi_{2}}{2}} h_{2} & \sqrt{\frac{\phi_{2}}{2}} h_{1}\end{array}\right.$} & Eq.(8) \\
\hline
\end{tabular}

$$
\begin{aligned}
\operatorname{Pr}\left(C_{1}^{\mathrm{AS}}<2 R\right)=\operatorname{Pr}\left(\log \left(1+\phi_{1} g_{1}\right)<2 R\right)=1-e^{-\frac{\theta}{\phi_{1}}}, \theta \doteq e^{2 R}-1, & \\
\operatorname{Pr}\left(C_{2}^{\mathrm{AS}}<R\right)=\operatorname{Pr}\left(\phi_{1} g_{1}+\phi_{2} g_{2}<\theta\right) & =\int_{0}^{\frac{\theta}{\phi_{1}}} f_{g_{1}}(x) \operatorname{Pr}\left(g_{2}<\frac{\theta-\phi_{1} x}{\phi_{2}}\right) \mathrm{d} x \\
& = \begin{cases}1-\frac{\phi_{1}}{\phi_{1}-\phi_{2}} e^{-\frac{\theta}{\phi_{1}}}-\frac{\phi_{2}}{\phi_{2}-\phi_{1}} e^{-\frac{\theta}{\phi_{2}}}, & \text { if } \phi_{1} \neq \phi_{2} \\
1-e^{-\frac{\theta}{\phi_{1}}}-\frac{\theta}{\phi_{1}} e^{-\frac{\theta}{\phi_{1}}}, & \text { if } \phi_{1}=\phi_{2} .\end{cases}
\end{aligned}
$$

SMR-base STC-ARQ:

$$
\begin{aligned}
& \operatorname{Pr}\left(C_{1}^{\mathrm{SMR}}<2 R\right)=\operatorname{Pr}\left(G \leq \frac{2 \theta}{\phi_{1}}\right)=\int_{0}^{\frac{2 \theta}{\phi_{1}}} f_{G}(x) \mathrm{d} x=1-e^{-\frac{2 \theta}{\phi_{1}}}-\frac{2 \theta}{\phi_{1}} e^{-\frac{2 \theta}{\phi_{1}}}, \\
& \operatorname{Pr}\left(C_{2}^{\mathrm{SMR}}<R\right)=\operatorname{Pr}\left(G \leq \frac{2 \theta}{\phi_{1}+\phi_{2}}\right)=1-e^{-\frac{2 \theta}{\phi_{1}+\phi_{2}}}-\frac{2 \theta}{\phi_{1}+\phi_{2}} e^{-\frac{2 \theta}{\phi_{1}+\phi_{2}}} .
\end{aligned}
$$

Alamouti-based STC-ARQ:

$$
\begin{aligned}
& \operatorname{Pr}\left(C_{1}^{\text {Alamouti }}<2 R\right)=\operatorname{Pr}\left(G \leq \frac{2 \theta}{\phi_{1}}\right)=1-e^{-\frac{2 \theta}{\phi_{1}}}-\frac{2 \theta}{\phi_{1}} e^{-\frac{2 \theta}{\phi_{1}}}, \\
& \operatorname{Pr}\left(C_{2}^{\text {Alamouti }}<R\right)=\operatorname{Pr}\left(\sum_{i=1}^{2} \log \left(1+\frac{\phi_{i}}{2} G\right) \leq 2 R\right) \stackrel{(a)}{=} \operatorname{Pr}(G \leq \sigma)=1-(1+\sigma) e^{-\sigma}, \\
& \sigma \doteq-\left(\frac{1}{\phi_{1}}+\frac{1}{\phi_{2}}\right)+\sqrt{\left(\frac{1}{\phi_{1}}+\frac{1}{\phi_{2}}\right)^{2}+\frac{4 \theta}{\phi_{1} \phi_{2}}} .
\end{aligned}
$$

Here, $(a)$ follows from the fact that $\theta \geq 0, G \geq 0$ and, as a result, the equation $\left(1+\frac{\phi_{1}}{2} G\right)\left(1+\frac{\phi_{2}}{2} G\right)=e^{2 R}$ has a single positive solution $G=\sigma$.

CDD-base STC-ARQ:

$$
\begin{aligned}
& \operatorname{Pr}\left(C_{1}^{\mathrm{CDD}}<2 R\right)=1-e^{-\frac{2 \theta}{\phi_{1}}}-\frac{2 \theta}{\phi_{1}} e^{-\frac{2 \theta}{\phi_{1}}} \\
& \operatorname{Pr}\left(C_{2}^{\mathrm{CDD}}<R\right)=\operatorname{Pr}\left(\left(1+\frac{\phi_{1}}{2} G\right)\left(1+\frac{\phi_{2}}{2} G\right)-\phi_{1} \phi_{2} \operatorname{Re}\left\{h_{1} h_{2}^{*}\right\}^{2} \leq e^{2 R}\right)
\end{aligned}
$$

while, to the best of authors' knowledge, the probability $\operatorname{Pr}\left(C_{2}^{\mathrm{CDD}}<R\right)$ can not be further simplified.

Observation 1. From (9)-(12), it is found that for all considered STCs we have $\operatorname{Pr}\left(C_{2}^{\mathrm{A}}<R\right)=\Omega_{2}^{\mathrm{A}}\left(\phi_{1}, \phi_{2}\right), \mathrm{A}=$ $\{$ AS, Alamouti, SMR, CDD $\}$, where $\Omega_{2}^{\mathrm{A}}\left(\phi_{1}, \phi_{2}\right)$ is a symmetric function of $\phi_{1}$ and $\phi_{2}$, i.e., the power terms $\phi_{1}$ and $\phi_{2}$, are interchangeable in $\Omega_{2}^{\mathrm{A}}\left(\phi_{1}, \phi_{2}\right)$. Also, $\operatorname{Pr}\left(C_{1}^{\mathrm{A}}<2 R\right)=\Omega_{1}^{\mathrm{A}}\left(\phi_{1}\right)$ which is a function of $\phi_{1}$ only. 
As a direct consequence of Observation 1, it is found that for all considered STCs the minimum energy-limited outage probability of the STC-based schemes, i.e., the solution of (5), is achieved by uniform power allocation $\phi_{1}=\phi_{2}=$ $\frac{\bar{\phi}}{2}, \bar{\phi}=\frac{\bar{\psi}}{L}$. This is because both the objective function and the constraint of (5) are symmetric functions of $\phi_{1}$ and $\phi_{2}$.

To determine the energy-limited minimum outage probability of the considered STC-ARQ protocols, i.e., solving (4), we write the Lagrange multiplier function

$$
\Upsilon^{\mathrm{A}}=\phi_{1}+\phi_{2} \Omega_{1}^{\mathrm{A}}\left(\phi_{1}\right)+\lambda^{\mathrm{A}} \Omega_{2}^{\mathrm{A}}\left(\phi_{1}, \phi_{2}\right)
$$

where $\lambda^{\mathrm{A}}$ is the Lagrange multiplier satisfying $\phi_{1}+\phi_{2} \Omega_{1}^{\mathrm{A}}\left(\phi_{1}\right)=\bar{\phi}^{\text {STC-ARQ }}$ for the STC-ARQ scheme A. Setting the derivatives with respect to $\phi_{1}$ and $\phi_{2}$ equal to zero leads to

$$
\left\{\begin{array}{l}
\frac{\partial \Upsilon^{\mathrm{A}}}{\partial \phi_{1}}=1+\phi_{2} \frac{\partial \Omega_{1}^{\mathrm{A}}}{\partial \phi_{1}}+\lambda^{\mathrm{A}} \frac{\partial \Omega_{2}^{\mathrm{A}}}{\partial \phi_{1}}=0, \\
\frac{\partial \Upsilon^{\mathrm{A}}}{\partial \phi_{2}}=\Omega_{1}^{\mathrm{A}}+\lambda^{\mathrm{A}} \frac{\partial \Omega_{2}^{\mathrm{A}}}{\partial \phi_{2}}=0,
\end{array} \quad \Rightarrow 1+\phi_{2} \frac{\partial \Omega_{1}^{\mathrm{A}}}{\partial \phi_{1}}=\Omega_{1}^{\mathrm{A}} \frac{\partial \Omega_{2}^{\mathrm{A}}}{\partial \phi_{1}}\left(\frac{\partial \Omega_{2}^{\mathrm{A}}}{\partial \phi_{2}}\right)^{-1} .\right.
$$

Thus, using $\phi_{2}=\frac{\bar{\phi}^{\text {STC-ARQ }}-\phi_{1}}{\Omega_{1}^{A}\left(\phi_{1}\right)}$ from (4.ii), the optimal power allocation rule of different STC-ARQ protocols are found as the solution of

$$
\left\{\begin{array}{l}
\hat{\phi}_{1}=\underset{\phi_{1}}{\arg }\left\{1+\left(\frac{\bar{\phi}^{\mathrm{STC}-A R Q}-\phi_{1}}{\Omega_{1}^{\mathrm{A}}}\right) \frac{\partial \Omega_{1}^{\mathrm{A}}}{\partial \phi_{1}}-\Omega_{1}^{\mathrm{A}}\left(\left.\frac{\partial \Omega_{2}^{\mathrm{A}}}{\partial \phi_{1}}\right|_{\phi_{2}=\frac{\bar{\phi}^{\mathrm{STC}-A R Q_{-}}}{\Omega_{1}^{\mathrm{A}}}}\right)\left(\left.\frac{\partial \Omega_{2}^{\mathrm{A}}}{\partial \phi_{2}}\right|_{\phi_{2}=\frac{\bar{\phi}^{S T C-A R Q}-\phi_{1}}{\Omega_{1}^{\mathrm{A}}}}\right)^{-1}=0\right\} \\
\hat{\phi}_{2}=\frac{\bar{\phi}^{\mathrm{STC}-\mathrm{ARQ}}-\hat{\phi}_{1}}{\Omega_{1}^{\mathrm{A}}\left(\hat{\phi}_{1}\right)}
\end{array}\right.
$$

where $\left.\frac{\partial \Omega_{2}^{\mathrm{A}}}{\partial \phi_{i}}\right|_{\phi_{2}=\frac{\bar{\phi}_{\mathrm{STC}-\mathrm{ARQ}}-\phi_{1}}{\Omega_{1}^{\mathrm{A}}}}$ denotes rewriting $\frac{\partial \Omega_{2}^{\mathrm{A}}}{\partial \phi_{i}}$ as a function of $\phi_{1}$ via (4.ii). Also, $\hat{\phi}_{1}, \hat{\phi}_{2}$ are the optimal values of powers, in terms of (4). Note that (15.i) is a single-variable equation from which $\hat{\phi}_{1}$ is derived. For instance, as $\frac{\partial \Omega_{1}^{\mathrm{SMR}}}{\partial \phi_{1}}=\frac{-4 \theta^{2}}{\phi_{1}^{3}} e^{-\frac{2 \theta}{\phi_{1}}}$ and $\frac{\partial \Omega_{2}^{\mathrm{SSR}}}{\partial \phi_{1}}=\frac{\partial \Omega_{2}^{\mathrm{SMR}}}{\partial \phi_{2}}=\frac{-4 \theta^{2}}{\left(\phi_{1}+\phi_{2}\right)^{3}} e^{-\frac{2 \theta}{\phi_{1}+\phi_{2}}}$, the optimal powers of the SMR-ARQ approach are given by

$$
\left\{\begin{array}{l}
\hat{\phi}_{1}=\underset{\phi_{1}}{\arg }\left\{e^{-\frac{2 \theta}{\phi_{1}}}=\frac{1+\frac{\phi_{1}^{2}}{4 \theta^{2}}+\frac{\phi_{1}}{2 \theta}-\frac{\bar{\phi}^{\mathrm{STC} C A R Q}}{\phi_{1}}}{\left(\frac{\phi_{1}}{2 \theta}+1\right)^{2}}\right\} \\
\hat{\phi}_{2}=\frac{\bar{\phi}^{\mathrm{STC}-\mathrm{ARQ}}-\hat{\phi}_{1}}{1-\left(1+\frac{2 \theta}{\bar{\phi}_{1}}\right) e^{-\frac{2 \theta}{\phi_{1}}}} .
\end{array}\right.
$$

Then, having $\hat{\phi}_{1}, \hat{\phi}_{2}$ the outage probability is calculated (The same procedure is applied for the other STCs ${ }^{2}$ ). Specially, it is interesting to obtain the optimal powers of different protocols at moderate/high SNRs, which is the range of interest in outage-limited conditions [5], [6], [9]. The results are summarized in Theorem 1.

Theorem 1. Consider the moderate/high SNRs and the optimization problem (4). Then, the optimal powers of the AS-ARQ protocol are given by $\left(\hat{\phi}_{1}, \hat{\phi}_{2}\right)=\left(\frac{2 \bar{\phi}^{\text {STCARQ }}}{3}, \frac{2 \bar{\phi}^{\text {STC-ARQ }}{ }^{2}}{9 \theta}\right)$. Also, the Alamouti-, the SMR- and the CDD-based STC-ARQ protocols follow the same optimal power allocation rule $\left(\hat{\phi}_{1}, \hat{\phi}_{2}\right)=\left(\frac{2 \bar{\phi}^{\mathrm{STC}-\mathrm{ARQ}}}{3}, \frac{2 \bar{\phi}^{\mathrm{STC}-\mathrm{ARQ}}{ }^{3}}{27 \theta^{2}}\right)$.

${ }^{2} \mathrm{As} \Omega_{2}^{\mathrm{CDD}}$ does not have closed-form expression based on, e.g., $f_{g_{i}}$ 's, the optimal powers of the CDD-ARQ should be derived via approaximation/bounding techniques, as illustrated in the following. 
Proof. Using $1-e^{\frac{-1}{x}}=\frac{1}{x}$ and $e^{\frac{-1}{x}}=1-\frac{1}{x}+\frac{1}{2 x^{2}}$ in (9.i) and (9.ii), respectively, and because $\phi_{1} \neq \phi_{2}$ when optimizing the power terms in the STC-ARQ protocols, we have $\Omega_{1}^{\mathrm{AS}}=\frac{\theta}{\phi_{1}}$ and $\Omega_{2}^{\mathrm{AS}}=\frac{\theta^{2}}{2 \phi_{1} \phi_{2}}$ for high values of $\phi_{1}, \phi_{2}$. Thus, implementing the AS-ARQ at moderate/high SNRs, (4) is rephrased as

$$
\left\{\begin{array} { l } 
{ \operatorname { m i n } _ { \phi _ { 1 } , \phi _ { 2 } } \frac { \theta ^ { 2 } } { 2 \phi _ { 1 } \phi _ { 2 } } , } \\
{ \text { s.t. } \phi _ { 1 } + \frac { \phi _ { 2 } \theta } { \phi _ { 1 } } = \overline { \phi } ^ { \mathrm { STC } - \mathrm { ARQ } } }
\end{array} \equiv \left\{\begin{array}{l}
\max _{\phi_{1}, \phi_{2}} \phi_{1} \phi_{2} \\
\text { s.t. } \phi_{2}=\frac{\left(\bar{\phi}^{\mathrm{STC}-A R Q}-\phi_{1}\right) \phi_{1}}{\theta}
\end{array}\right.\right.
$$

which follows from the fact that $\Omega_{2}^{\mathrm{AS}}=\frac{\theta^{2}}{2 \phi_{1} \phi_{2}}$ is a decreasing function of the product $\phi_{1} \phi_{2}$. Solving (17), the high-SNR power allocation rule of the AS-ARQ scheme is found as $\left(\hat{\phi}_{1}, \hat{\phi}_{2}\right)=\left(\frac{2 \bar{\phi}^{\text {STC-ARQ }}}{3}, \frac{2 \bar{\phi}^{\text {STCARQ }^{2}}}{9 \theta}\right)$, as stated in the theorem. The optimal powers of the other protocols are obtained with the same procedure; using the first-order Taylor expansion of the exponential terms in (10), we have $\Omega_{1}^{\mathrm{SMR}}=\frac{2 \theta^{2}}{\phi_{1}^{2}}$ and $\Omega_{2}^{\mathrm{SMR}}=\frac{2 \theta^{2}}{\left(\phi_{1}+\phi_{2}\right)^{2}}$ at high SNRs, which rephrases (4) as

$$
\left\{\begin{array} { l } 
{ \operatorname { m i n } _ { \phi _ { 1 } , \phi _ { 2 } } \frac { 2 \theta ^ { 2 } } { ( \phi _ { 1 } + \phi _ { 2 } ) ^ { 2 } } , } \\
{ \text { s.t. } \phi _ { 1 } + \frac { 2 \phi _ { 2 } \theta ^ { 2 } } { \phi _ { 1 } ^ { 2 } } = \overline { \phi } ^ { \text { STC-ARQ } } }
\end{array} \equiv \left\{\begin{array}{l}
\max _{\phi_{1}, \phi_{2}}\left(\phi_{1}+\phi_{2}\right) \\
\text { s.t. } \phi_{2}=\frac{\left(\bar{\phi}^{\text {STC-ARQ }}-\phi_{1}\right) \phi_{1}^{2}}{2 \theta^{2}}
\end{array}\right.\right.
$$

Hence, the optimal power allocation rule of the SMR-ARQ protocol is obtained by $\frac{\partial\left(\phi_{1}+\frac{\left(\bar{\phi}^{\mathrm{STC}-A R Q}-\phi_{1}\right) \phi_{1}^{2}}{2 \theta^{2}}\right)}{\partial \phi_{1}}=0$ which, ignoring its lowest term at high SNRs, results in $\left(\hat{\phi}_{1}, \hat{\phi}_{2}\right)=\left(\frac{2 \bar{\phi}^{\mathrm{STCARQ}}}{3}, \frac{2 \bar{\phi}^{\mathrm{STC}-\mathrm{ARQ}}{ }^{3}}{27 \theta^{2}}\right)$.

For the Alamouti coding, we note that $\Omega_{1}^{\text {Alamouti }}=\frac{2 \theta^{2}}{\phi_{1}^{2}}$ at high SNRs. Also,

$$
\Omega_{2}^{\text {Alamouti }}=1-(1+\sigma) e^{-\sigma} \stackrel{(b)}{=} \frac{\sigma^{2}}{2}=\frac{1}{2}\left(\frac{1}{\phi_{1}}+\frac{1}{\phi_{2}}\right)^{2}\left(-1+\sqrt{1+\frac{4 \theta \phi_{1} \phi_{2}}{\left(\phi_{1}+\phi_{2}\right)^{2}}}\right)^{2} \stackrel{(c)}{=} \frac{2 \theta^{2}}{\left(\phi_{1}+\phi_{2}\right)^{2}} .
$$

Here, $(b)$ follows from $e^{-\sigma}=1-\sigma+\frac{\sigma^{2}}{2}$ for small $\sigma$ 's (note that $\sigma \rightarrow 0$ as $\bar{\phi}^{\text {STC-ARQ }} \rightarrow \infty$ ) and $(c)$ is obtained by $\sqrt{1+x}=1+\frac{x}{2}$ as $x \rightarrow 0$. In this way, the optimal power terms of the Alamouti-based scheme are obtained by (18), the same as in the SMR, i.e., $\left(\hat{\phi}_{1}, \hat{\phi}_{2}\right)=\left(\frac{2 \bar{\phi}^{\text {STCARQ }}}{3}, \frac{2 \bar{\phi}^{\text {STCARQ }}{ }^{3}}{27 \theta^{2}}\right)$.

Finally, for the CDD-ARQ scheme, we note 1) the SMR- and the Alamouti-based schemes follow the same power allocation rules at high SNRs, 2) $\Omega_{1}^{\mathrm{SMR}}=\Omega_{1}^{\mathrm{CDD}}=\Omega_{1}^{\text {Alamouti }}, \forall \phi_{1}$, and 3) using Table 1 it is straightforward to show that $C_{2}^{\mathrm{SMR}} \leq C_{2}^{\mathrm{CDD}} \leq C_{2}^{\text {Alamouti }}$ for every given set of $h_{1}, h_{2}, \phi_{1}, \phi_{2}$. Hence, the outage probabilities are ordered as

$$
\Omega_{2}^{\mathrm{SMR}} \geq \Omega_{2}^{\mathrm{CDD}} \geq \Omega_{2}^{\text {Alamouti }}, \forall R, \phi_{1}, \phi_{2}
$$

and, from (18)-(19), $\Omega_{2}^{\mathrm{SMR}}=\Omega_{2}^{\text {Alamouti }}=\frac{2 \theta^{2}}{\left(\phi_{1}+\phi_{2}\right)^{2}}$ at high SNRs. Thus, based on the Squeeze Theorem of limits [17, Chapter 14.2], the high-SNR optimal powers of the CDD-ARQ protocol are $\left(\hat{\phi}_{1}, \hat{\phi}_{2}\right)=\left(\frac{2 \bar{\phi}^{\mathrm{STC}-\mathrm{ARQ}}}{3}, \frac{2 \bar{\phi}^{\mathrm{STC}-\mathrm{ARQ}}{ }^{3}}{27 \theta^{2}}\right)$, the same as the Alamouti- and the SMR-ARQ schemes.

Intuitively, Theorem 1 indicates that at high SNRs the data is correctly decoded at the end of the first round with a very high probability. Thus, with a total energy budget, substantially high energy can be assigned to the second round, as it is rarely used. Also, as an interesting corollary, the theorem shows that, although the Alamouti-based (resp. the CDD-based) 
scheme outperforms the CDD-based (resp. the SMR-based) approach, in terms of outage probability, they lead to the same performance at high SNRs. To elaborate on this point and emphasize the effect of optimal power allocation, we obtain the diversity gain $D=-\lim _{\mathrm{SNR} \rightarrow \infty} \frac{\log (\operatorname{Pr}(\text { Outage }))}{\log \mathrm{SNR}}$ [9, eq. 14] of the considered schemes in Corollary 1. Particularly, the corollary shows that the implementation of power-optimized ARQ scales up the diversity gain of the AS, the Alamouti, the SMR and the CDD protocols from 2 to $3,6,6$ and 6 , respectively ${ }^{3}$.

Corollary 1. The diversity gains of the AS-, the Alamouti-, the SMR- and the CDD-based STC-ARQ protocols are $3,6,6$ and 6 , respectively, if the power terms are optimized in terms of (4).

Proof. Considering the high SNRs, i.e., $\bar{\phi}^{\mathrm{STC}-\mathrm{ARQ}} \rightarrow \infty$ in $(4)$, we have $\left(\hat{\phi}_{1}, \hat{\phi}_{2}\right)=\left(\frac{2 \bar{\phi}^{\mathrm{STC}-\mathrm{ARQ}}}{3}, \frac{2 \bar{\phi}^{\mathrm{STC} C \mathrm{ARQ}}{ }^{2}}{9 \theta}\right)$ for the AS-ARQ which, following the same arguments as in Theorem 1 , results in outage probability $\Omega_{2}^{\mathrm{AS}}=\frac{27 \theta^{3}}{8 \bar{\phi}^{\mathrm{STC}-\mathrm{ARQ}^{3}}}$ and

$$
D^{\mathrm{AS}-\mathrm{ARQ}}=-\lim _{\bar{\phi}^{\mathrm{STC}-\mathrm{ARQ}} \rightarrow \infty} \frac{\log \left(\frac{27 \theta^{3}}{8 \bar{\phi}^{\mathrm{TTC}-\mathrm{ARQ}} \mathrm{Q}^{3}}\right)}{\log \bar{\phi}^{\mathrm{STC}-\mathrm{ARQ}}}=3 .
$$

The same procedure is applied to derive the diversity gain for the other protocols; replacing $\left(\hat{\phi}_{1}, \hat{\phi}_{2}\right)=\left(\frac{2 \bar{\phi}^{\mathrm{STC}-\mathrm{ARQ}}}{3}, \frac{2 \bar{\phi}^{\mathrm{STC}-\mathrm{ARQ}}{ }^{3}}{27 \theta^{2}}\right)$ into the approximate expressions of the outage probability in (18)-(19), we have

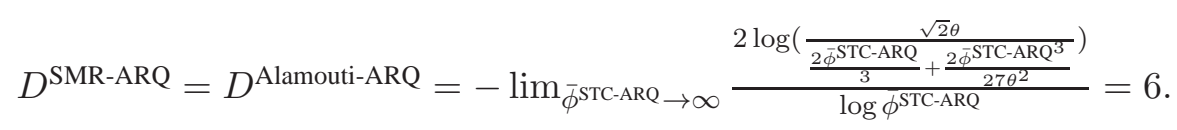

Finally, for the CDD-ARQ we reuse the Squeeze Theorem and (20) which leads to $D^{\mathrm{CDD}-\mathrm{ARQ}}=6$.

Setting $Q^{\mathrm{f}}=0$ and $R=1$ (npcu), Fig.1a shows the outage probability of different STC-ARQ protocols and compares the results with the ones achieved by the STC. As demonstrated, the implementation of ARQ improves the energy efficiency substantially. For instance, with an outage probability $10^{-4}$, the required transmission SNR, i.e., $\bar{\phi}$, of the AS, the Alamouti, the SMR and the CDD kinds of STC is decreased by 6.4, 8.7, 10.8 and $9.3 \mathrm{~dB}$, respectively, if they are combined with ARQ. Moreover, we can use, e.g., [6]-[8], [10], [14], and follow the same procedure as in (6)-(8) to show that, for a $2 \times 1$ MISO setup, $C_{i}^{\text {Type II }}=C_{i}^{\text {Alamouti }}$ and $C_{i}^{\text {Type III }}=C_{i}^{\text {SMR }}, \forall h_{1}, h_{2}, i=1,2$. Thus, with a maximum of $M=2$ retransmissions, the energy-limited outage probability of the Alamouti-ARQ (resp. SMR-ARQ) is the same as the one achieved by Type II [6] (resp. Type III [8]) ARQ, which is because $\operatorname{Pr}\left(C_{i}^{\text {Type II }} \leq x\right)=\operatorname{Pr}\left(C_{i}^{\text {Alamouti }} \leq x\right)$ and $\operatorname{Pr}\left(C_{i}^{\text {Type III }} \leq x\right)=\operatorname{Pr}\left(C_{i}^{\text {SMR }} \leq x\right), \forall x, i=1,2$. Finally, the figure emphasizes the validity of Theorem $1 /$ Corollary 1 , where the outage probability of different schemes follow the same order as in (20) and the diversity gains, i.e., the negative of the slope of the curves at high SNRs, matches the ones derived in Corollary 1.

Shown in Fig.1b are the optimal values of $\phi_{2}$ for the AS- and the Alamouti-ARQ protocols and the ones achieved through the approximation techniques of Theorem 1. For moderate/high SNR, the approximations match the exact values with very high accuracy.

\footnotetext{
${ }^{3}$ Following the same procedure as in Corollary 1, the diversity gain of all considered STCs (without ARQ) is found as $D=2$.
} 

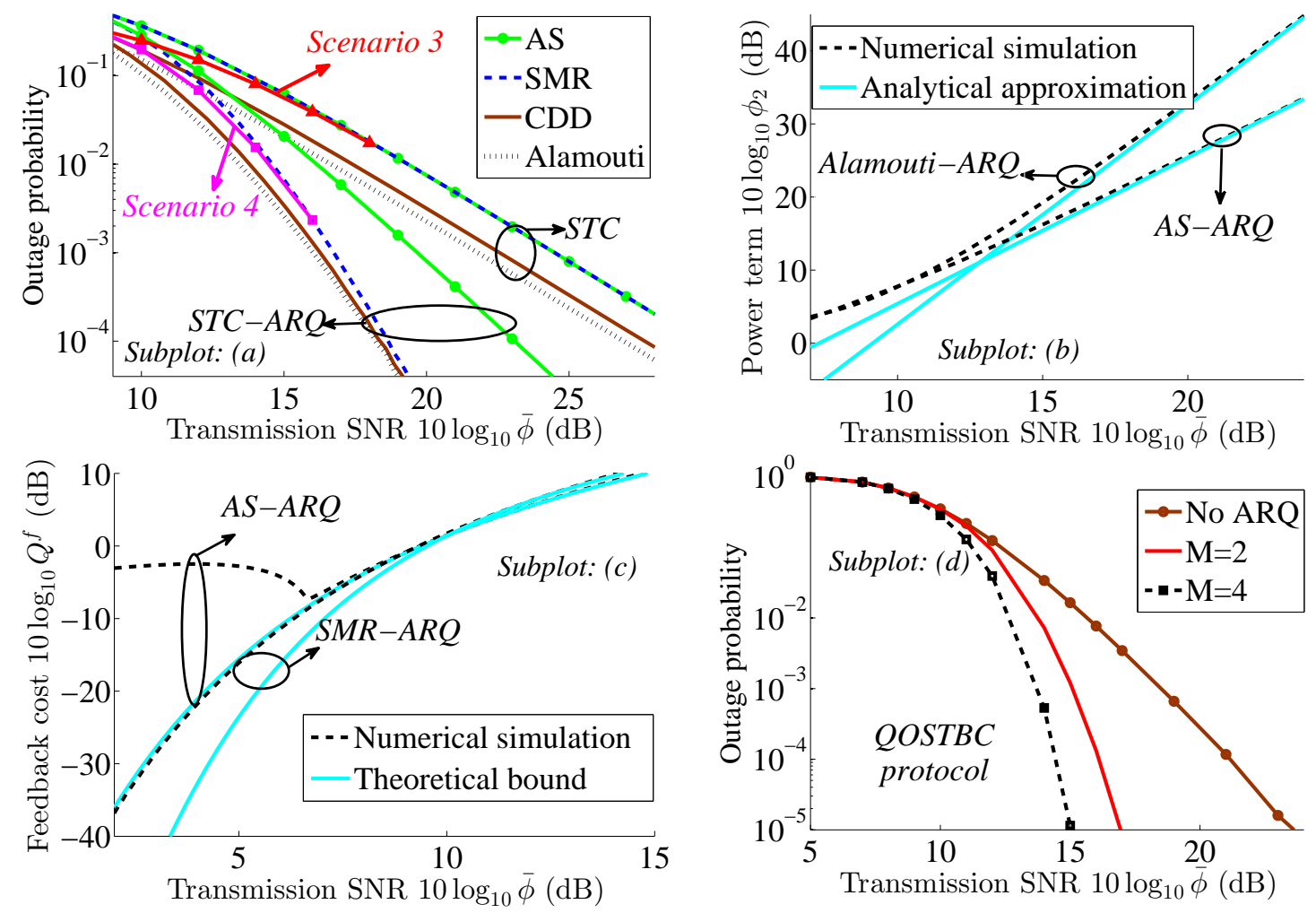

Figure 1. (a): Comparison between the STC, the STC-ARQ and the data transmission schemes of Scenarios 3-4. The same outage probability as in the Alamouti-ARQ (resp. SMR-ARQ) is achieved by Type II (resp. Type III) ARQ protocol. (b): Comparison between the optimal values of $\phi_{2}$ obtained via numerical simulations and the theoretical approximations of Theorem 1. (c): Acceptable range of relative feedback cost $Q^{\mathrm{f}}$ vs the transmission SNR $\bar{\phi}$. For the considered STCs, the bounds in Theorem 2 match the ones obtained via Corollary 2. (d): Outage probability for the QOSTBC-ARQ protocol with different partitionings of the mother codeword. In all figures, we set $R=1$ npcu. Figs.(a)-(c) are for a $2 \times 1$ MISO setup. Fig.(d) represents the results for a $4 \times 1$ MISO system. In Figs. (a), (b) and (d) the results are obtained for $Q^{\mathrm{f}}=0$.

Throughout the paper, we concentrate on the ARQ schemes, motivated by the fact that the ARQ is already implemented in most standards and implies low implementation complexity. However, the same procedure can be applied to combine the STC and other feedback approaches. For instance, let us consider the scenarios where the transmitter is informed via one bit channel state information (CSI) feedback at the beginning of the codeword transmission (resp. at the end of round 1) whether the channel is in outage, such that the whole codeword (resp. the second sub-codeword) should not be sent. In these models, referred to as Scenario 3 (resp. Scenario 4), the power allocation problem (4) is rephrased as Scenario 3: $\left\{\begin{array}{l}\min _{\phi_{1}, \phi_{2}} \operatorname{Pr}\left(C_{2}<R\right), \\ \text { s.t. }\left(\phi_{1}+\phi_{2}\right) \operatorname{Pr}\left(C_{2}>R\right) \leq \bar{\phi}-Q^{\mathrm{f}},\end{array}\right.$ Scenario 4: $\left\{\begin{array}{l}\min _{\phi_{1}, \phi_{2}} \operatorname{Pr}\left(C_{2}<R\right), \\ \text { s.t. } \phi_{1}+\phi_{2} \operatorname{Pr}\left(C_{1}<2 R \& C_{2}>R\right) \leq \bar{\phi}-Q^{\mathrm{f}} .\end{array}\right.$ Fig.1a shows the performance of these scenarios for the SMR STC. As seen, at moderate/high SNRs, on which we focus, the same performance as in the STC (resp. STC-ARQ) is achieved in Scenario 3 (resp. Scenario 4). Thus, depending on the complexity/available feedback resources, one can consider different feedback approaches. Finally, it is straightforward to prove that the diversity gain and the high-SNR optimal power allocation of Scenario 3 (resp. Scenario 4) are the same 
as the ones in Scenario 2 (resp. Scenario 1) (For further comparison between different feedback schemes, see [7]).

\section{A. On the Effect of Feedback Cost}

As mentioned before, the implementation of ARQ improves the energy efficiency of a STC scheme if the expected gain achieved by sending only part of the mother codeword exceeds the feedback cost. To investigate the range of feedback costs for which the STC-ARQ approach outperforms the original STC, in terms of energy-limited outage probability, one can derive the optimal power allocation rules of these schemes and compare their outage probability for each kind of STC. The procedure is as follows. For every given value of $\bar{\phi}$, uniform power allocation and (5) are used to determine the minimum outage probability of the considered STC. Then, considering (4), we sweep on different values of relative feedback costs $Q^{\mathrm{f}}$ in the range of $[0, \bar{\phi}]$ and follow the same procedure as in (15) to find the maximum value of $Q^{\mathrm{f}}$ for which the STC-ARQ leads to less outage probability, compared to the STC-based scheme.

The following Theorem demonstrates a sufficient condition for the usefulness of the ARQ.

Theorem 2. The AS-based (resp. the Alamouti-, the SMR- and the CDD-based) STC-ARQ outperforms the STC scheme, in terms of energy-limited outage probability, if $Q^{\mathrm{f}} \leq \frac{\bar{\phi}}{2} e^{\frac{-2 \theta}{\bar{\phi}}}\left(\right.$ resp. $\left.Q^{\mathrm{f}} \leq \frac{\bar{\phi}}{2}\left(1+\frac{4 \theta}{\bar{\phi}}\right) e^{-\frac{4 \theta}{\bar{\phi}}}\right)$.

Proof. As shown before, the minimum outage probability of all considered (non-ARQ) STC schemes is achieved by $\phi_{1}=\phi_{2}=\frac{\bar{\phi}}{2}$. Let us consider the same power for the first round of the STC-ARQ protocol, i.e., $\phi_{1}=\frac{\bar{\phi}}{2}$, which is not necessarily optimal for the STC-ARQ protocol. As the outage probability is a decreasing function of transmission power $\phi_{2}$, the STC-ARQ leads to less outage probability, compared to the STC scheme, if $\phi_{2}>\frac{\bar{\phi}}{2}$. Moreover, using (4.ii) and $\phi_{1}=\frac{\bar{\phi}}{2}$, we have

$$
\phi_{2}=\frac{\frac{\bar{\phi}}{2}-Q^{\mathrm{f}}}{\operatorname{Pr}\left(C_{1}^{\mathrm{A}}<2 R\right)}, \mathrm{A}=\{\text { Alamouti, AS, CDD, SMR }\} .
$$

Thus, considering the constraint $\phi_{2}>\frac{\bar{\phi}}{2}$, a sufficient condition for the usefulness of ARQ, i.e., a lower bound on the acceptable range of feedback costs, is found as $\frac{1-\frac{2 Q^{\mathrm{f}}}{\phi}}{\operatorname{Pr}\left(C_{1}^{\mathrm{A}}<2 R\right)} \geq 1$ which, using (9)-(12) and $\phi_{1}=\frac{\bar{\phi}}{2}$, leads to

$$
Q^{\mathrm{f}} \leq \begin{cases}\frac{\bar{\phi}}{2} e^{\frac{-2 \theta}{\phi}}, & \text { for AS-STC } \\ \frac{\bar{\phi}}{2}\left(1+\frac{4 \theta}{\phi}\right) e^{-\frac{4 \theta}{\phi}}, & \text { for Alamouti-, CDD-, SMR-STC. }\end{cases}
$$

Considering the AS and the SMR kinds of STC, Fig.1c shows the acceptable range of feedback costs versus the SNR. Each curve in Fig.1c shows the set of feedback costs for which the ARQ is useful improving the energy efficiency of the STC-based systems. This set of feedback costs corresponds to the area below each curve. Thus, the higher the curve is, the higher feedback cost is tolerated. Also, the figure compares the numerical results with the bounds developed in Theorem 2. As it is seen, 1) the proposed bounds are very tight at moderate/high SNRs. 2) At low SNRs, the AS-ARQ tolerates higher feedback costs, compared to the SMR (and the Alamouti/CDD-ARQ, although not demonstrated in the figure). However, the acceptable range of feedback costs of the considered schemes converges at high SNRs. 


\section{B. Extension of Results to Other Kinds of STC/Network Configurations}

Throughout the paper, we concentrated on the $2 \times 1$ MISO setup and the results where obtained for the AS, the Alamouti, the SMR and the CDD kinds of STC. Our reasons for selecting the $2 \times 1$ MISO setup are 1) the analytical results can be easily explained/followed, and 2) the STCs have been first designed for the $2 \times 1 \mathrm{MISO}$ channel. However, the same procedure as the one presented in Sections II-III can be applied for the cases with more number of antennas/other kinds of STC. Specially, partitioning the mother codeword of a STC approach into $M$ equal-length sub-codewords and sending the sub-codewords in an ARQ-based fashion, the weighted energy and the outage probability are obtained by

$$
\begin{aligned}
& \Psi^{\text {STC-ARQ }}=L\left(\sum_{i=1}^{M} \phi_{i} \Omega_{i-1}+Q^{\mathrm{f}} \sum_{i=0}^{M-2} \Omega_{i}\right), \Omega_{0} \doteq 1, \\
& \operatorname{Pr}(\text { Outage })=\Omega_{M}, \Omega_{m} \doteq \operatorname{Pr}\left(C_{m}<\frac{M R}{m}\right),
\end{aligned}
$$

respectively, where $\Omega_{m}$ is the probability that the message is not correctly decoded in round $m$. Then, deriving the achievable rate terms $C_{m}$, one can follow the same iterative derivation approach as in (15) or use other optimization algorithms such as [7, Algorithm 1], to find the optimal powers, in terms of energy-limited outage probability. Indeed, with more number of antennas or other kinds of STC the mother codeword can be broken into sub-codewords in several ways, and the proper partitioning of the mother codeword can be determined based on, e.g., the feedback cost. As an example, considering a $4 \times 1 \mathrm{MISO}$ setup and $Q^{\mathrm{f}}=0$, Fig.1d shows the performance of the quasi-orthogonal space-time block code (QOSTBC) [13, eq. 2] in the cases with a maximum of $M=2$ and 4 (re)transmissions, i.e., partitioning the QOSTBS mother codeword into 2 and 4 equal-length sub-codewords, respectively (see [13, eq. 2] for the mother codeword of the QOSTBC). As it can be seen, the combination of ARQ and STC reduces the outage probability considerably, and the effect of ARQ increases by increasing the maximum number of retransmissions $M$.

We close the discussions with the following corollary that extends the results of Theorem 2 to different ARQ schemes.

Corollary 2. For every STC-ARQ protocol, a sufficient condition for the usefulness of ARQ, in terms of energy-limited outage probability, is given by $\frac{Q^{\mathrm{f}}}{\phi} \leq \frac{(M-1)(1-\epsilon)}{M(1+(M-2) \epsilon)}$, where $\epsilon$ is the outage probability in round 1 with $\phi_{1}=\frac{\bar{\phi}}{M}$.

Proof. The proof follows the same trend as in Theorem 2; set $\phi_{i}=\frac{\bar{\phi}}{M}, \forall i$, for the STC-ARQ protocol which is not necessarily optimal, in terms of energy-limited outage probability. Therefore, the same outage probability is achieved by the STC and the STC-ARQ schemes. From (25.i) and $\phi_{i}=\frac{\bar{\phi}}{M}, \forall i$, the STC-ARQ approach leads to less total weighted energy, compared to the STC, if

$$
\frac{\bar{\phi}}{M} \sum_{i=1}^{M} \Omega_{i-1}+Q^{\mathrm{f}} \sum_{i=0}^{M-2} \Omega_{i} \leq \bar{\phi} \Leftrightarrow Q^{\mathrm{f}} \leq \bar{\phi}\left(\frac{1-\frac{1}{M} \sum_{i=1}^{M} \Omega_{i-1}}{\sum_{i=0}^{M-2} \Omega_{i}}\right) \stackrel{(d)}{\Leftrightarrow} \frac{Q^{\mathrm{f}}}{\bar{\phi}} \leq \frac{(M-1)(1-\epsilon)}{M(1+(M-2) \epsilon)} .
$$

Here, $(d)$ is obtained by some manipulations and the fact that $\Omega_{0}=1$ and $\Omega_{i} \leq \epsilon, \forall i$.

For $M=2$ and different STCs, the bound (26) matches the ones presented in Theorem 2. Also, the simulations show the tightness of the bound at high SNR (Fig.1c). Finally, in harmony with Fig.1c, (26) emphasizes that the ARQ feedback 
cost is of more importance at low SNRs, i.e., $\epsilon \approx 1$, while its effect decreases at low values of $\epsilon$, i.e., high SNRs.

\section{CONCLUSION}

This correspondence studied the effect of adaptive power allocation on the performance of the STC-based systems utilizing ARQ. As demonstrated, the energy-limited outage probability of the STC-ARQ setups is reduced substantially if the sub-codewords of the STC are scaled appropriately. Specially, optimal power allocation increases the diversity gain of the STC-ARQ protocols remarkably. Finally, the joint design of ARQ and STC achieves considerable energy efficiency gain for a large range of ARQ feedback costs.

\section{REFERENCES}

[1] B. Makki, T. Svensson, and M. Zorzi, “Green communication via Type-I ARQ: Finite block-length analysis,” in Globecom, 2014, accepted.

[2] D. V. Djonin, A. K. Karmokar, and V. K. Bhargava, "Joint rate and power adaptation for type-I hybrid ARQ systems over correlated fading channels under different buffer-cost constraints," IEEE Trans. Veh. Technol., vol. 57, no. 1, pp. 421-435, Jan. 2008.

[3] M. K. Chang, C. S. Lim, and C. C. J. Kuo, "Power control for packet-based wireless communication systems," in WCNC, vol. 1, March 2003, pp. 542-546.

[4] H. Seo and B. G. Lee, "Optimal transmission power for single- and multi-hop links in wireless packet networks with ARQ capability," IEEE Trans. Commun., vol. 55, no. 5, pp. 996-1006, May 2007.

[5] P. Wu and N. Jindal, "Performance of hybrid-ARQ in block-fading channels: A fixed outage probability analysis," IEEE Trans. Commun., vol. 58, no. 4, pp. 1129-1141, April 2010.

[6] T. V. K. Chaitanya and E. G. Larsson, “Outage-optimal power allocation for hybrid ARQ with incremental redundancy," IEEE Trans. Wireless Commun., vol. 10, no. 7, pp. 2069-2074, July 2011.

[7] B. Makki and T. Eriksson, "On hybrid ARQ and quantized CSI feedback schemes in quasi-static fading channels," IEEE Trans. Commun., vol. 60, no. 4, pp. 986-997, April 2012.

[8] I. Stanojev, O. Simeone, Y. Bar-Ness, and D. H. Kim, "Energy efficiency of non-collaborative and collaborative hybrid-ARQ protocols," IEEE Trans. Wireless Commun., vol. 8, no. 1, pp. 326-335, Jan. 2009.

[9] H. El-Gamal, G. Caire, and M. O. Damen, "The MIMO ARQ channel: Diversity-multiplexing-delay tradeoff," IEEE Trans. Inf. Theory, vol. 52, no. 8, pp. 3601-3621, Aug. 2006.

[10] C. Shen and M. P. Fitz, "Hybrid ARQ in multiple-antenna slow fading channels: Performance limits and optimal linear dispersion code design,” IEEE Trans. Inf. Theory, vol. 57, no. 9, pp. 5863-5883, Sept. 2011.

[11] M. Gidlund, "Design and performance of space-time block coded hybrid ARQ schemes for multiple antenna transmission," in ISPACS, Feb. 2009, pp. 1-4.

[12] A. L. Toledo and X. Wang, "TCP performance over wireless MIMO channels with ARQ and packet combining," IEEE Trans. Mobile Comput., vol. 5, no. 3, pp. 208-223, March 2006.

[13] X. Lu, G. Zhu, and L. Liu, G. Li, “A cross-layer design over MIMO Rayleigh fading channels,” in WiCOM, vol. 1, 2005 , pp. 50-53.

[14] B. Makki and T. Eriksson, "On the performance of MIMO-ARQ systems with channel state information at the receiver," IEEE Trans. Commun., vol. 62, no. 5, pp. 1588-1603, May 2014.

[15] A. Ghosh, D. R. Wolter, J. G. Andrews, and R. Chen, "Broadband wireless access with WiMax/802.16: current performance benchmarks and future potential," IEEE Commun. Mag., vol. 43, no. 2, pp. 129-136, Feb. 2005.

[16] E. Telatar, "Capacity of multi-antenna Gaussian channels," Bell Labs, Tech. Rep., June 1995.

[17] J. Stewart, Multivariable calculus. Cengage Learning, 2011. 